\title{
ON THE PROBLEM OF SYMMETRIZATION OF HYPERBOLIC EQUATIONS
}

\author{
V. I. KOSTIN \\ Institute of Mathematics, Russian Academy of Sciences, Siberian Branch \\ Universitetski亢 Prosp. 4, 630090 Novosibirsk, Russia
}

Introduction. The aspects of symmetrization of hyperbolic equations which will be considered in this review have their own history and are related to some classical results from other areas of mathematics ([12]). Here symmetrization means representation of an initial system of equations in the form of a symmetric $t$-hyperbolic system in the sense of Friedrichs. Some equations of mathematical physics, for example, the equations of acoustics, of gas dynamics, etc. already have this form. In the 70's S. K. Godunov published a work [8] on a symmetric form of the equations of magnetohydrodynamics. This result was repeated in the 80 's ([3]). Later A. M. Blokhin ([1]) got an analogous result for the Landau equations of quantum helium. All the mentioned statements concern systems of equations describing concrete physical objects.

One of the motivations for investigating the symmetrization problem comes from the study of initial-boundary value problems for hyperbolic equations. Having a rich set of energy integrals for a given hyperbolic equation one can use them to get estimates of solutions in the well posed problems. Generally one uses a fairly simple theory of initial-boundary value problems with dissipative boundary conditions (see e.g. [7]). This idea has been realized in some simplest cases ([2, $10,11,18])$.

2. Statement of the symmetrization problem. There are different ways to formalize the problem of energy integrals for hyperbolic equations. We give one that concerns linear operators and goes back to Leray's constructions ([6, 13 , 17]). First we recall

Definition 1. A linear differential operator

$$
P\left(t, x ; D_{t}, D_{x}\right)=\sum_{i+|r| \leq n} p_{i r}(t, x) D_{t}^{i} D_{x}^{r}
$$


of order $n$ is called (strictly) t-hyperbolic (in the sense of Petrovskiu) in the domain $\Omega$ if for all $(t, x) \in \Omega$ and for each real $\xi(|\xi| \neq 0)$ the characteristic polynomial

$$
P(t, x ; \tau, \xi)=\sum_{i+|r|=n} p_{i r}(t, x) \tau^{i} \xi^{r}
$$

has $n$ distinct real roots

$$
\tau_{1}(t, x ; \xi)<\ldots<\tau_{n}(t, x ; \xi) .
$$

(If not stated otherwise, we use everywhere the vector notations

$$
x=\left(x_{1}, \ldots, x_{m}\right), \quad D_{x}=\left(D_{x_{1}}, \ldots, D_{x_{m}}\right), \quad \xi=\left(\xi_{1}, \ldots, \xi_{m}\right),
$$

and $r=\left(r_{1}, \ldots, r_{m}\right)$ is a multi-index.)

There exists another notion of hyperbolicity ([5]):

Definition 2. A matrix linear first order differential operator

$$
A(t, x) D_{t}+\sum_{j=1}^{m} B_{j}(t, x) D_{x_{j}}+Q(x, t)
$$

is called symmetric t-hyperbolic (in the sense of Friedrichs) in $\Omega$ if for all $(t, x) \in$ $\Omega$ the matrices $A=A(t, x), B_{j}=B_{j}(t, x)$ are symmetric (hermitian) $\left(A^{*}=\right.$ $\left.A, B_{j}^{*}=B_{j}\right)$ and $A$ is positive definite $(A>0)$.

For solutions $U(t, x)$ of the symmetric hyperbolic system

$$
\left[A(t, x) D_{t}+\sum_{j=1}^{m} B_{j}(t, x) D_{x_{j}}+Q(x, t)\right] U(t, x)=F(t, x)
$$

the so-called differential identity for the energy integral is well known:

$$
\begin{array}{r}
D_{t}(A(t, x) U(t, x), U(t, x))+\sum_{j=1}^{m} D_{x_{j}}\left(B_{j}(t, x) U(t, x), U(t, x)\right) \\
+\left(\left[Q(t, x)+Q^{*}(t, x)-D_{t} A(t, x)-\sum_{j=1}^{m} D_{x_{j}} B_{j}(t, x)\right] U(t, x), U(t, x)\right) \\
=2 \operatorname{Re}(F(t, x), U(t, x)) .
\end{array}
$$

(Here and below $(\cdot, \cdot)$ denotes the inner product of column vectors. This notation is also used for bilinear forms on vectors.)

Notice that in the case of Definition 2 for real $\xi \neq 0$ all the roots $\tau_{j}(t, x ; \xi)$ of the characteristic equation

$$
\operatorname{det}\left[A(t, x) \tau+\sum_{j=1}^{m} B_{j}(t, x) \xi_{j}\right]=0
$$

are real but they may be multiple. 
In his work [5] Friedrichs showed how to construct a symmetric hyperbolic system from a second order hyperbolic equation, that is, in our terms he symmetrized the second order equation. In his construction the components of the unknown vector function are the first partial derivatives of a function $u(t, x)$ which is a solution of the initial equation. Because of the importance and great clarity of Friedrichs' construction we repeat it here but using our formal technique. From now on we only consider operators with constant coefficients. The standard technique generalizes the results to the case of variable coefficients [15]. Also, we consider operators including only principal terms:

$$
P\left(D_{t}, D_{x}\right)=\sum_{i+|r|=n} p_{i r} D_{t}^{i} D_{x}^{r}
$$

Thus the characteristic polynomial $P(\tau, \xi)=\sum p_{i r} \tau^{i} \xi^{r}$ is homogeneous of degree $n$.

Now we recall the Friedrichs construction. Let $P(\tau, \xi)=\tau^{2}+2 \tau \sum a_{j} \xi_{j}+$ $\sum b_{i j} \xi_{i} \xi_{j}$ be the characteristic polynomial of a second order hyperbolic operator. We will just call it a hyperbolic polynomial. The hyperbolicity condition from Definition 1 leads to

$$
\left(\sum a_{j} \xi_{j}\right)^{2}-\sum b_{i j} \xi_{i} \xi_{j}>0 \quad \text { for } \sum \xi_{j}^{2} \neq 0 .
$$

For simplicity we assume a more restrictive condition:

$$
\sum b_{i j} \xi_{i} \xi_{j}<0 \quad \text { for } \sum \xi_{j}^{2} \neq 0 .
$$

In the terminology used below, this means that the polynomial $\tau$ of degree one is a partitioning polynomial for $P(\tau, \xi)$. The following identity is easy to verify:

$$
\begin{aligned}
& \left(\tau\left[\begin{array}{cccc}
1 & 0 & \ldots & 0 \\
0 & b_{11} & \ldots & b_{1 m} \\
\ldots & \ldots & \ldots & \ldots \\
0 & b_{m 1} & \ldots & b_{m m}
\end{array}\right]+\xi_{1}\left[\begin{array}{cccc}
2 a_{1} & b_{11} & \ldots & b_{1 m} \\
b_{11} & 0 & \ldots & 0 \\
\ldots \ldots & \ldots & \ldots & \ldots \\
b_{m 1} & 0 & \ldots & 0
\end{array}\right]+\ldots\right. \\
& \left.+\xi_{m}\left[\begin{array}{cccc}
2 a_{m} & b_{m 1} & \ldots & b_{m m} \\
b_{1 m} & 0 & \ldots & 0 \\
\ldots \ldots & \ldots \ldots \ldots . . . \\
b_{m m} & 0 & \ldots & 0
\end{array}\right]\right)\left[\begin{array}{c}
\tau \\
\xi_{1} \\
\vdots \\
\xi_{m}
\end{array}\right]=\left[\begin{array}{c}
P(\tau, \xi) \\
0 \\
\vdots \\
0
\end{array}\right] .
\end{aligned}
$$

Set

$$
A=\left[\begin{array}{cccc}
1 & 0 & \ldots & 0 \\
0 & b_{11} & \ldots & b_{1 m} \\
\ldots & \ldots & \ldots & \ldots . . \\
0 & b_{m 1} & \ldots & b_{m m}
\end{array}\right], \quad B_{j}=\left[\begin{array}{cccc}
2 a_{j} & b_{j 1} & \ldots & b_{j j} \\
b_{1 j} & 0 & \ldots & 0 \\
\ldots \ldots & \ldots & \ldots & \ldots \\
b_{m j} & 0 & \ldots & 0
\end{array}\right]
$$

and $r(\tau, \xi)=\left(\tau, \xi_{1}, \ldots, \xi_{m}\right)^{T}, g=(1,0, \ldots, 0)^{T}$. Then we can write $(2.4)$ in the 
form

$$
\left(\tau A+\sum_{j=1}^{m} \xi_{j} B_{j}\right) r(\tau, \xi)=P(\tau, \xi) g
$$

It follows that

$$
\left(A D_{t}+\sum_{j=1}^{m} B_{j} D_{x_{j}}\right) r\left(D_{t}, D_{x}\right) u(t, x)=P\left(D_{t}, D_{x}\right) u(t, x) g,
$$

where $u(t, x)$ is an arbitrary sufficiently smooth function. Thus if $u(t, x)$ is a solution of $P\left(D_{t}, D_{x}\right) u=f(t, x)$ then the vector function $U(t, x)=\left(u_{t}, u_{x_{1}}, \ldots\right.$ $\left.\ldots, u_{x_{m}}\right)^{T}$ satisfies the first order system

$$
\left(A D_{t}+\sum_{j=1}^{m} B_{j} D_{x_{j}}\right) U(t, x)=F(t, x),
$$

where $F(t, x)=f(t, x) g$. The matrices of this system are symmetric and $A$ is positive definite by $(2.3)$.

For brevity the symmetric system (2.6) will be called the symmetrization of the equation $P\left(D_{t}, D_{x}\right) u=f(t, x)$. This symmetrization practically coincides with the construction in [5]. The polynomial identity constructed was fundamental here; the system of differential equations (2.6) follows from (2.5) in a trivial way.

Notice that if $\xi \neq 0$ and $\tau=\tau(\xi)$ is a root of the characteristic equation $P(\tau, \xi)=0$ then from $(2.5)$ we have

$$
\left(\tau A+\sum_{j=1}^{m} \xi_{j} B_{j}\right) r(\tau, \xi)=0 .
$$

As the vector $r(\tau, \xi)$ is nonzero, $\operatorname{det}\left(\tau A+\sum \xi_{j} B_{j}\right)=0$ for those values of $\tau, \xi$. Thus the roots of the characteristic polynomial $P(\tau, \xi)$ are the roots of the characteristic polynomial $\operatorname{det}\left(\tau A+\sum \xi_{j} B_{j}\right)$. In other words, the characteristic cone of the initial equation is embedded in the characteristic cone of its symmetrization.

Below we consider a family of symmetrizations of a hyperbolic operator depending on several parameters and such that the above property of characteristic polynomials is valid for any symmetrization. Naturally, a problem arises to describe those symmetrizations for which the Hamilton-Jacobi cones of the initial operator and of its symmetrization coincide (for definition see, for example, [7]). The answer was obtained by T. Yu. Mikhailova ([19]) for rotation invariant operators.

From the polynomial identities (2.5) we can get another identity playing an important role below. For this purpose let us multiply both sides of (2.5) scalarly by the vector $r(\lambda, \alpha)$ :

$$
\tau(A r(\tau, \xi), r(\lambda, \alpha))+\sum_{j=1}^{m} \xi_{j}\left(B_{j} r(\tau, \xi), r(\lambda, \alpha)\right)=P(\tau, \xi) \lambda .
$$


Notice that (2.5) follows from (2.7) if we equate the coefficients of the variables $\lambda, \alpha_{1}, \ldots, \alpha_{m}$ on both sides of (2.7).

Now let us write the identity symmetric to (2.7), obtained by substituting $\tau \rightarrow \lambda, \lambda \rightarrow \tau, \xi_{j} \rightarrow \alpha_{j}, \alpha_{j} \rightarrow \xi_{j}:$

$$
\lambda(A r(\lambda, \xi), r(\tau, \xi))+\sum_{j=1}^{m} \alpha_{j}\left(B_{j} r(\lambda, \alpha), r(\tau, \xi)\right)=P(\lambda, \alpha) \tau .
$$

Adding (2.7) and (2.8) and using the symmetry of the matrices $A, B_{j}$, we get the so-called Hörmander identity for the polynomials $P(\tau, \xi)$ and $\tau$ :

$$
\begin{aligned}
P(\tau, \xi) \lambda & +P(\lambda, \alpha) \tau \\
& =(\tau+\lambda)(A r(\tau, \xi), r(\lambda, \alpha))+\sum_{j=1}^{m}\left(\xi_{j}+\alpha_{j}\right)\left(B_{j} r(\tau, \xi), r(\lambda, \alpha)\right) .
\end{aligned}
$$

In the next section (2.9) will be generalized to the case of higher order operators with any number of variables.

To finish this section we give another construction for an arbitrary hyperbolic polynomial in two variables $\tau, \xi(m=1)$. Let $P(\tau, \xi)=\prod_{j=1}^{N}\left(\tau-c_{j} \xi\right)$ be a hyperbolic polynomial (the $c_{j}$ are real and pairwise distinct). For definiteness we suppose that $c_{1}<\ldots<c_{N}$. It is trivial to verify the identity

$$
\left(\tau\left[\begin{array}{ccc}
1 & & 0 \\
& \ddots & \\
0 & & 1
\end{array}\right]+\xi\left[\begin{array}{ccc}
-c_{1} & & 0 \\
& \ddots & \\
0 & & -c_{N}
\end{array}\right]\right)\left[\begin{array}{c}
p_{1}(\tau, \xi) \\
\vdots \\
p_{N}(\tau, \xi)
\end{array}\right]=P(\tau, \xi)\left[\begin{array}{c}
1 \\
\vdots \\
1
\end{array}\right]
$$

where $p_{j}(\tau, \xi)=P(\tau, \xi) /\left(\tau-c_{j} \xi\right)$. Here the matrices are symmetric and the matrix attached to $\tau$ is positive definite.

If we suppose that the values of the differential operators $p_{j}\left(D_{t}, D_{x}\right)$ on a scalar function $u(t, x)$ are the components of a vector function $U(t, x)$, then a symmetric hyperbolic system for that vector function follows immediately from (2.10). The matrices of this system are equal to the matrices in (2.10). However, using the linear independence of the polynomials $p_{j}(\tau, \xi)$ in the space of homogeneous polynomials in $\tau, \xi$ of degree $N-1$ we can use the standard monomial basis $\tau^{N-1}, \tau^{N-2} \xi, \tau^{N-3} \xi^{2}, \ldots, \xi^{N-1}$. If $\mathbf{P}$ is the transition matrix then we get

$$
(\tau A+\xi B) r(\tau, \xi)=\mathbf{P}(\tau, \xi) g
$$

where

$$
\begin{gathered}
A=\mathbf{P}^{*} \mathbf{P}, \quad B=\mathbf{P}^{*} \operatorname{diag}\left(-c_{1}, \ldots,-c_{N}\right) \mathbf{P}, \\
r(\tau, \xi)=\left(\tau^{N-1}, \tau^{N-2} \xi, \tau^{N-3} \xi^{2}, \ldots, \xi^{N-1}\right)^{T}, \\
g=\mathbf{P}^{*}(1, \ldots, 1)^{T} .
\end{gathered}
$$

Multiplying both sides of (2.10) by the vector

$$
\left(p_{1}(\lambda, \alpha), \ldots, p_{N}(\lambda, \alpha)\right)^{T}
$$


and adding the resulting equality to a symetric one we get the Hörmander identity in this case:

$$
(\tau+\lambda) F[\tau, \xi ; \lambda, \alpha]+(\xi+\alpha) G[\tau, \xi ; \lambda, \alpha]=P(\tau, \xi) Q(\lambda, \alpha)+Q(\tau, \xi) P(\lambda, \alpha) .
$$

The following notations are used here:

$$
\begin{aligned}
F[\tau, \xi ; \lambda, \alpha] & =\sum p_{j}(\tau, \xi) p_{j}(\lambda, \alpha), \\
G[\tau, \xi ; \lambda, \alpha] & =-\sum c_{j} p_{j}(\tau, \xi) p_{j}(\lambda, \alpha), \\
Q(\tau, \xi) & =\sum p_{j}(\tau, \xi) .
\end{aligned}
$$

It is easily seen that

$$
F[\tau, \xi ; \lambda, \alpha]=F[\lambda, \alpha ; \tau, \xi], \quad G[\tau, \xi ; \lambda, \alpha]=G[\lambda, \alpha ; \tau, \xi]
$$

and these polynomials are homogeneous of degree $N-1$ separately with respect to both groups of variables $(\tau, \xi)$ and $(\lambda, \alpha)$. Moreover, $Q(\tau, \xi)=(d / d \tau) P(\tau, \xi)$ and so the roots $\tau_{j}(\xi)=d_{j} \xi(j=1, \ldots, N-1)$ of $Q$ separate the roots of $P$, i.e.

$$
c_{1}<d_{1}<c_{2}<d_{2}<c_{3}<\ldots<c_{N-1}<d_{N-1}<c_{N} .
$$

3. The Hörmander identity. At the beginning of this section (in contrast to the previous notations) we will not express the variable corresponding to the derivative with respect to $t$ in operators and will not assume the hyperbolicity of polynomials. Let $P(\xi)=P\left(\xi_{1}, \ldots, \xi_{m}\right)$ and $Q(\xi)$ be homogeneous polynomials of degree $N$ and $N-1$, respectively. The Hörmander identity is an expansion

$$
P(\xi) Q(\alpha)+Q(\xi) P(\alpha)=\sum_{j=1}^{m}\left(\xi_{j}+\alpha_{j}\right) G_{j}[\xi ; \alpha],
$$

where $\alpha=\left(\alpha_{1}, \ldots, \alpha_{m}\right)$ and the polynomials $G_{j}[\xi ; \alpha]$ are hermitian and homogeneous:

$$
G_{j}[\xi ; \alpha]=\overline{G_{j}[\alpha ; \xi]}
$$

and for any real scalar $\varrho$,

$$
G_{j}[\varrho \xi ; \alpha]=G_{j}[\xi ; \varrho \alpha]=\varrho^{N-1} G_{j}[\xi ; \alpha] .
$$

Notice that (3.1) implies the identity $P(\xi) Q(\alpha)=\sum_{j} \xi_{j} G_{j}[\xi ; \alpha]$, which in turn yields

$$
\sum_{j} \xi_{j} A^{[j]} r(\xi)=P(\xi) g .
$$

Here $r(\xi)$ is the column of all homogeneous monomials $r_{i}(\xi)$ of degree $N-1$ in the variables $\left(\xi_{1}, \ldots, \xi_{m}\right), g$ is the column of coefficients of $Q(\xi)$ expanded with respect to the monomials, and the elements of the matrices $A^{[j]}$ are the coefficients 
of the polynomial $G_{j}[\xi ; \alpha]$ :

$$
G_{j}[\xi ; \alpha]=\sum_{i, k} a_{i k}^{[j]} r_{i}(\xi) r_{k}(\alpha) .
$$

Obviously, the above-mentioned properties of the polynomials $G_{j}[\xi ; \alpha]$ ensure the matrices $A^{[j]}$ in (3.4) to be symmetric. The Hörmander identity will be our principal tool below.

Some difficulties are connected with the nonuniqueness of the polynomials $G_{j}[\xi ; \alpha]$ defined by the above conditions. In other words, (3.1) considered as an equation for $G_{j}$ has a general solution $G_{j}^{n}[\xi ; \alpha]+G_{j}^{h}[\xi ; \alpha]$, where $G_{j}^{n}[\xi ; \alpha]$ is some particular solution of (3.1) and $G_{j}^{h}[\xi ; \alpha]$ satisfies the homogeneous equation

$$
\sum_{j=1}^{m}\left(\xi_{j}+\alpha_{j}\right) G_{j}^{h}[\xi ; \alpha]=0 .
$$

An algorithm for constructing a particular solution $G_{j}^{n}[\xi ; \alpha]$ is given in [15] (see also [9]) for the case $m=3$. The solution of the homogeneous identity (3.5) in this case was also described there. In the general case, the equation (3.5) was investigated in [21] where all solutions of (3.1), or, which is the same, all matrices $A^{[j]}$ in (3.4), were described.

Other difficulties arise when we try to get symmetrizations with a positive definite matrix attached to the operator $D_{t}$ in Friedrichs' definition. Now we explain the nature of those difficulties. It will now be convenient to distinguish the variable corresponding to the operator $D_{t}$ in the initial differential operator $P\left(D_{t}, D_{x}\right)$. From now on we assume that $P$ is $t$-hyperbolic in the sense of Petrovskiri, i.e. for real $\xi \neq 0$ the homogeneous polynomial $P(\tau, \xi)$ of degree $N$ has real and distinct roots

$$
\tau_{1}(\xi)<\ldots<\tau_{N}(\xi) .
$$

These roots are homogeneous functions in $\xi$ of degree one. The Hörmander identity is

$$
\begin{aligned}
P(\tau, \xi) Q(\lambda, \alpha)+Q(\tau, \xi) & P(\lambda, \alpha) \\
& =(\tau+\lambda) F[\tau, \xi ; \lambda, \alpha]+\sum_{j}\left(\xi_{j}+\alpha_{j}\right) G[\tau, \xi ; \lambda, \alpha],
\end{aligned}
$$

and in the new notations (3.4) reads

$$
\left(\tau A+\sum_{j=1}^{m} \xi_{j} B_{j}\right) r(\tau, \xi)=P(\tau, \xi) g .
$$

We confine ourselves to polynomials $Q(\tau, \xi)$ partitioning for $P(\tau, \xi)$. By definition, this means that for real $\xi \neq 0$ the roots $\mu_{j}(\xi)$ of $Q(\mu, \xi)$ satisfy the inequalities

$$
\tau_{1}(\xi)<\mu_{1}(\xi)<\tau_{2}(\xi)<\mu_{2}(\xi)<\ldots<\mu_{N-1}(\xi)<\tau_{N}(\xi) .
$$


The reason for that is explained by the following fact. If the form $F[\tau, \xi ; \lambda, \xi]$ obtained from $F[\tau, \xi ; \lambda, \alpha]$ by identifying $\xi$ and $\alpha$ is expanded with respect to powers of $\tau$ and $\lambda$ :

$$
F[\tau, \xi ; \lambda, \xi]=\sum_{i, j=1}^{N} f_{i j}(\xi) \tau^{N-i-1} \lambda^{N-j-1}
$$

then the symmetric matrix of order $N$

$$
\mathcal{F}(\xi)=\left(f_{i j}(\xi)\right)_{i, j=1}^{N}
$$

is positive definite for real $\xi \neq 0$. This surprising fact directly follows from the Hermite theorem (see, for example, $[16,6,13,17]$ ) and from the simple equality

$$
F[\tau, \xi ; \lambda, \xi]=\frac{P(\tau, \xi) Q(\lambda, \xi)-P(\lambda, \xi) Q(\tau, \xi)}{\tau-\lambda},
$$

which may be obtained from (3.7) by setting $\alpha=-\xi$ and using homogeneity.

Thus the trace of the form $F[\tau, \xi ; \lambda, \alpha]$ corresponds to a positive definite matrix depending on parameters. The matrix (3.11) is uniquely determined by the polynomials $P(\tau, \xi)$ and $Q(\tau, \xi)$. The nonuniqueness in "solutions" of the Hörmander identity leads to the nonuniqueness of the matrix $A$ to be determined in a symmetrization and, as a corollary, to impossibility of $A>0$ for all solutions $\left(F, G_{j}\right)$ of (3.7). It turns out that in symmetrization only the form $F[\tau, \xi ; \lambda, \alpha]$ is important if it has a true trace. This fact is formulated as the next lemma.

LEMma. Let $\left(F^{n}, G^{n}\right)$ be a "solution" of the Hörmander identity (3.7), and let $\mathcal{F}[\tau, \xi ; \lambda, \alpha]$ be an arbitrary homogeneous symmetric form equal to $F^{n}$ for $\xi=\alpha$. Then there exist forms $\mathcal{G}_{j}[\tau, \xi ; \lambda, \alpha]$ such that $\left(\mathcal{F}[\tau, \xi ; \lambda, \alpha], \mathcal{G}_{j}[\tau, \xi ; \lambda, \alpha]\right)$ satisfies (3.7).

There is an interesting connection with an old problem posed first by D. Hilbert ([12]). Consider (3.10) for $\lambda=\tau$. We get $F[\tau, \xi ; \tau, \xi]>0$ for $\tau \neq 0, \xi \neq 0$ because $\mathcal{F}(\xi)>0$. Suppose that we have found a positive definite (real for simplicity) matrix $A$ such that

$$
F[\tau, \xi ; \lambda, \alpha]=\sum_{i, j} a_{i j} r_{i}(\tau, \xi) r_{j}(\tau, \xi)
$$

where $\left\{r_{i}(\tau, \xi)\right\}$ is the basis of all monomials of degree $N-1$ in the variables $\tau, \xi$. Expanding $A$ in the form $B^{*} B$ we can write

$$
\begin{aligned}
F[\tau, \xi ; \lambda, \alpha] & =(A r(\tau, \xi), r(\lambda, \alpha))=(B r(\tau, \xi), B r(\lambda, \alpha)) \\
& =\sum_{i}\left(\sum_{j} b_{i j} r_{j}(\tau, \xi)\right) \cdot\left(\sum_{j} b_{i j} r_{j}(\lambda, \alpha)\right) .
\end{aligned}
$$

Let $\tau=\lambda, \xi=\alpha$ in (3.14). Then

$$
F[\tau, \xi ; \tau, \xi]=\sum_{i}\left(\sum_{j} b_{i j} r_{j}(\tau, \xi)\right)^{2} .
$$


Thus we have written the positive definite form $F[\tau, \xi ; \tau, \xi]$ as a sum of squares. As noticed by D. Hilbert, this is not possible for every such form. Though in our case $F[\tau, \xi ; \tau, \xi]$ is not an arbitrary positive definite form because it is obtained from the polynomials $P(\tau, \xi)$ and $Q(\tau, \xi)$ by means of $(3.12)$, the possibility described by Hilbert can appear for such forms (see Section 5 ). In other words, a pair of hyperbolic polynomials not always gives birth to a symmetrization, and all difficulties are connected with guaranteeing the positive definiteness of the matrix $A$ in (3.8).

\section{Symmetrization: The case of three variables}

TheOREM $([16,19])$. Hyperbolic polynomials $P\left(\tau, \xi_{1}, \xi_{2}\right)$ in three variables are always symmetrizable.

The symmetrization is constructed in the following way. For all real $\xi$, the matrix $\mathcal{F}(\xi)$ of $(3.11)$ may be represented as the sum $\mathcal{F}^{[1]}(\xi)+\mathcal{F}^{[2]}(\xi)$ of two positive definite matrices, with $\mathcal{F}^{[1]}(\xi)$ diagonal. Its diagonal elements are

$$
f_{i i}^{[1]}=\varrho\left(\xi_{1}^{2}+\xi_{2}^{2}\right)^{i-1} \quad(i=1, \ldots, N)
$$

and the parameter $\varrho$ is chosen so small that $\mathcal{F}^{[2]}(\xi)>0$. Notice that the polynomial $\sum_{i=1}^{N} \varrho\left(\xi_{1}^{2}+\xi_{2}^{2}\right)^{i-1} \tau^{N-i-1} \lambda^{N-i-1}$ corresponds to the form $\sum_{i=1}^{N} \varrho\left(\xi_{1} \alpha_{1}+\right.$ $\left.\xi_{2} \alpha_{2}\right)^{i-1} \tau^{N-i-1} \lambda^{N-i-1}$ and it is the trace of this form for $\xi_{1}=\alpha_{1}, \xi_{2}=\alpha_{2}$. It is easily seen that the last form may be represented as $\left(A^{[1]} r(\tau, \xi), r(\lambda, \alpha)\right)$ with the diagonal matrix $A^{[1]}>0$.

Further, for $\xi_{1}=\alpha_{1}, \xi_{2}=\alpha_{2}$ the trace of $F[\tau, \xi ; \lambda, \alpha]-\left(A^{[1]} r(\tau, \xi), r(\lambda, \alpha)\right)$ may be represented as $\sum f_{i j}^{[2]}(\xi) \tau^{N-i-1} \lambda^{N-j-1}$ with a positive definite coefficient matrix $\mathcal{F}^{[2]}(\xi)$.

We need the following theorem.

Theorem ([20]). If $D(\varphi)=\sum_{k=-K}^{K} e^{i k \varphi} D^{[k]}$ is a hermitian trigonometric matrix polynomial positive definite for all real $\varphi$, then it may be represented as a product $D(\varphi)=\Delta^{*}(\varphi) \cdot \Delta(\varphi)$, where $\Delta(\varphi)=\sum_{k=0}^{K} e^{i k \varphi} \Delta^{[k]}$ is a trigonometric matrix polynomial. The polynomial $\Delta(\varphi)$ may be chosen to satisfy the following conditions: $\operatorname{det}\left[\sum_{k=0}^{K} \zeta^{k} \Delta^{[k]}\right] \neq 0$ for all complex $|\zeta| \leq 1$ and $\Delta^{[0]}$ is a hermitian positive definite matrix. $\Delta(\varphi)$ is uniquely determined by these conditions.

With the help of this theorem and taking into consideration the structure of the matrix elements of $\mathcal{F}^{[2]}(\xi)$ we can represent this matrix in the form $\mathcal{B}^{*}(\xi) \mathcal{B}(\xi)$ with a polynomial matrix $\mathcal{B}(\xi)$. For all $i$, the elements $b_{i j}(\xi)$ of this matrix are homogeneous (possibly complex) polynomials in $\xi$ of degree $j$. This leads to the representation (cf. (3.10))

$$
F^{[2]}[\tau, \xi ; \lambda, \xi]=\sum_{i, j=1}^{N} \sum_{k=1}^{N-1} \overline{b_{k i}(\xi)} \tau^{N-i-1} b_{k j}(\xi) \lambda^{N-j-1} .
$$


Consequently,

$$
F[\tau, \xi ; \lambda, \alpha]=(A r(\tau, \xi), r(\lambda, \alpha)),
$$

where $A=A^{[1]}+B^{*} B>0$ and the elements of $B$ are expressed through those of $\mathcal{B}(\xi)$. Here the matrix $B$ is rectangular and therefore $B^{*} B \geq 0$ (the rank of the product $B^{*} B$ equals $\left.N\right)$. We stress that namely this circumstance prevents us from using Rosenblatt's theorem at once for the matrix $\mathcal{F}(\xi)$, because then we will only get $A \geq 0$.

This construction is entirely elementary and it permits us to describe all possible symmetrizations of a hyperbolic polynomial $P\left(\tau, \xi_{1}, \xi_{2}\right)$ using a family of parameters determined by its partitioning polynomials and a "solution" of the homogeneous Hörmander identity

$$
(\tau+\lambda) F^{h}[\tau, \xi ; \lambda, \alpha]+\left(\xi_{1}+\alpha_{1}\right) G_{1}^{h}[\tau, \xi ; \lambda, \alpha]+\left(\xi_{2}+\alpha_{2}\right) G_{2}^{h}[\tau, \xi ; \lambda, \alpha]=0 .
$$

The "solution" in this case has the following form:

$$
\begin{aligned}
F^{h}[\tau, \xi ; \lambda, \alpha] & =\left(\xi_{1} \alpha_{2}-\xi_{2} \alpha_{1}\right) K[\tau, \xi ; \lambda, \alpha], \\
G_{1}^{h}[\tau, \xi ; \lambda, \alpha] & =\left(\xi_{2} \lambda-\alpha_{2} \tau\right) K[\tau, \xi ; \lambda, \alpha], \\
G_{2}^{h}[\tau, \xi ; \lambda, \alpha] & =\left(\tau \xi_{1}-\lambda \alpha_{1}\right) K[\tau, \xi ; \lambda, \alpha],
\end{aligned}
$$

where $K[\tau, \xi ; \lambda, \alpha]$ is a homogeneous form of degree $N-2$ with respect to the groups of variables $\left(\tau, \xi_{1}, \xi_{2}\right),\left(\lambda, \alpha_{1}, \alpha_{2}\right)$, which is also skew-hermitian $((3.2),(3.3))$.

5. Symmetrization: more than 3 variables. In this case the situation is analogous to that described in [12], i.e. there exist symmetrizable and nonsymmetrizable polynomials, and both sets of polynomials have a nonempty interior in the space of all homogeneous polynomials. To demonstrate this we show that the polynomials

$$
P(\tau, \xi)=\prod_{j=1}^{N}\left(\tau^{2}-c_{j}^{2}|\xi|^{2}\right), \quad P(\tau, \xi)=\tau \prod_{j=1}^{N}\left(\tau^{2}-c_{j}^{2}|\xi|^{2}\right)
$$

are symmetrizable if $c_{1}<\ldots<c_{N}$. These are the characteristic polynomials for products of wave operators with distinct velocities.

We only consider one case of even degree in (5.1) and we suppose that the partitioning polynomial has the same type (for another approach see [9]):

$$
Q(\tau, \xi)=\tau \prod_{j=1}^{N-1}\left(\tau^{2}-f_{j}^{2}|\xi|^{2}\right)
$$

It is obvious that

$$
c_{1}<f_{1}<c_{2}<f_{2}<c_{3}<\ldots<c_{N-1}<f_{N-1}<c_{N} .
$$

As mentioned in Section 3 the principal difficulties in symmetrization lie in the proof of the positive definiteness of the matrix $A$ in the identities (3.8) with sym- 
metric matrices. In the considered case this difficulty is overcome in the following way.

When $\xi \neq 0$ the polynomial

$$
F[\tau, \lambda ; \xi]=\frac{P(\tau, \xi) Q(\lambda, \xi)-P(\lambda, \xi) Q(\tau, \xi)}{\tau-\lambda}
$$

determines the positive definite matrix

$$
\mathcal{F}(\xi)=\left(f_{i j}(\xi)\right)_{i, j=1}^{N}
$$

(cf. (3.11)) and its elements are functions of $|\xi|^{2}$. (We hope that the change of notations will not lead to misunderstanding - the notation $F[\tau, \lambda ; \xi]$ seems to be more appropriate in the present case.) As in the previous section we can write $F[\tau, \lambda ; \xi]$ as the sum of two forms $F^{[1]}[\tau, \lambda ; \xi]+F^{[2]}[\tau, \lambda ; \xi]$, where

$$
F^{[1]}[\tau, \lambda ; \xi]=\varepsilon \sum_{i} \lambda^{i-1} \tau^{i-1}|\xi|^{4 N-2 i}=\sum_{i, j} a_{i j}^{[1]} r_{i}(\tau, \xi) r_{j}(\tau, \xi)
$$

and $\varepsilon>0$ is sufficiently small. The matrix $A^{[1]}$ of coefficients in (5.3) is diagonal and positive definite. Just as $F[\tau, \lambda ; \xi]$, the polynomial $F^{[2]}[\tau, \lambda ; \xi]$ determines a positive definite matrix $\mathcal{F}^{[2]}(\xi)$. It may be shown that $F^{[2]}[\tau, \lambda ; \xi]$ admits the following representation:

$$
\begin{aligned}
\sum_{p=1}^{N} \sum_{j=1}^{m}\left(\sum_{g=0}^{N-1} s_{p, 2 g+1}|\xi|^{2 N-2 g-2} \xi_{j} \tau^{2 g}\right)\left(\sum_{g=0}^{N-1} s_{p, 2 g+1}|\xi|^{2 N-2 g-2} \xi_{j} \tau^{2 g}\right) \\
+\sum_{p=N+1}^{2 N}\left(\sum_{h=0}^{N-1} s_{p, 2 h}|\xi|^{2 N-2 h} \tau^{2 h-1}\right)\left(\sum_{h=0}^{N-1} s_{p, 2 h}|\xi|^{2 N-2 h} \tau^{2 h-1}\right) .
\end{aligned}
$$

Therefore,

$$
F^{[2]}[\tau, \lambda ; \xi]=(B r(\tau, \xi), B r(\tau, \xi))
$$

where the column vector $r(\tau, \xi)$ consists of all monomials of degree $2 N-1$ in $m+1$ variables (the number of them equals $(2 N+m-1) ! /(2 N-1) ! m !)$ and $B$ is the matrix of coefficients in (5.4). The proof of (5.4) is based on the fact that the matrix elements of $\mathcal{F}^{[2]}(\xi)$ depend only on $|\xi|^{2}$. The coefficients $s_{i j}$ in (5.4) are the elements of a special matrix $S$ satisfying $S^{*} S=\mathcal{F}^{[2]}(1)$, where $\mathcal{F}(1)$ denotes the matrix $\mathcal{F}^{[2]}(\xi)$ for $|\xi|=1$.

Using (5.3), (5.5) we can write an expansion for $F[\tau, \xi ; \lambda, \alpha]$ :

$$
(A r(\tau, \xi), r(\lambda, \alpha))=\left(A^{[1]} r(\tau, \xi), r(\lambda, \alpha)\right)+(B r(\tau, \xi), B r(\lambda, \alpha))
$$

which for $\xi=\alpha$ coincides with $F[\tau, \lambda ; \xi]$ by construction. Now it remains to use the lemma from Section 3.

Using elementary continuity considerations it is easy to show that the hyperbolic polynomial $\widetilde{P}(\tau, \xi)$ sufficiently close to $(5.1)$ is symmetrizable. So the set of symmetrizable polynomials has a nonempty interior. 
As mentioned above, for $N \geq 4, m \geq 4$ there exist nonsymmetrizable polynomials of degree $N$ in $m$ variables. This was first shown by V. V. Ivanov ([14]). His proof uses the existence of positive definite homogeneous forms which cannot be expanded in a sum of squares. An example of such a form of degree 4 in 4 variables $\xi_{1}, \xi_{2}, \xi_{3}, \xi_{4}$ is (cf. [4])

$$
\begin{aligned}
q\left(\xi_{1}, \xi_{2}, \xi_{3}, \xi_{4}\right)= & \left(\xi_{1}^{4}+\xi_{2}^{2} \xi_{3}^{2}+\xi_{3}^{2} \xi_{4}^{2}+\xi_{4}^{2} \xi_{2}^{2}-4 \xi_{1} \xi_{2} \xi_{3} \xi_{4}\right) /(1+2 \varepsilon) \\
& +\varepsilon\left(\xi_{1}^{2}+\xi_{2}^{2}+\xi_{3}^{2}+\xi_{4}^{2}\right)^{2} /(1+2 \varepsilon)
\end{aligned}
$$

where $0<\varepsilon<1 / 4$ is a parameter. V. V. Ivanov proved that for any pair of polynomials $P, Q$ which lead to symmetrization in the scheme considered above, all the principal minors of the matrix $\mathcal{F}(\xi)$ in $(3.11)$ expand in a sum of squares. It is shown in [14] that for $0<\varepsilon<1 / 4$ the strictly hyperbolic polynomial

$$
P\left(\tau, \xi_{1}, \xi_{2}, \xi_{3}, \xi_{4}\right)=\left(\tau^{2}-\xi_{1}^{2}-\xi_{2}^{2}-\xi_{3}^{2}-\xi_{4}^{2}\right)^{2}-q\left(\xi_{1}, \xi_{2}, \xi_{3}, \xi_{4}\right)
$$

is nonsymmetrizable for any partitioning polynomial $Q\left(\tau, \xi_{1}, \xi_{2}, \xi_{3}, \xi_{4}\right)$ because the corresponding matrix $\mathcal{F}(\xi)$ has a principal minor which does not expand as a sum of squares whatever $Q$ is chosen. In other words, the matrix $A$ in (3.8) is not always positive definite.

\section{References}

[1] A. M. Blokhin, On symmetrization of the Landau equations in the theory of superfluidity of helium II, Dinamika Sploshn. Sredy 68 (1984), 13-34 (in Russian).

[2] -, Uniqueness of classical solution of gas dynamics mixed problem with boundary conditions on a shock wave, Sibirsk. Mat. Zh. 23 (5) (1982), 17-30 (in Russian).

[3] G. Boillat, Symmétrisation des systèmes d'équations aux dérivées partielles avec densité d'énergie convexe et contraintes, C. R. Acad. Sci. Paris Sér. I 295 (9) (1982), 551-554.

[4] M. D. Choi and T. Y. Lam, Extremal positive semidefinite forms, Math. Ann. 231 (1) (1977), 1-18.

[5] K. O. Friedrichs, Symmetric hyperbolic linear differential equations, Comm. Pure Appl. Math. 5 (2) (1954), 345-392.

[6] L. Gårding, Cauchy's Problem for Hyperbolic Equations, Chicago 1957.

[7] S. K. Godunov, Equations of Mathematical Physics, Nauka, Moscow 1979 (in Russian).

[8] -, A symmetric form of the equations of magnetohydrodynamics, Chisl. Metody Mekh. Sploshn. Sredy 3 (1) (1972), 26-34 (in Russian).

[9] S. K. Godunov and V. I. Kost in, Transformation of a hyperbolic equation to a symmetric hyperbolic system in the case of two spatial variables, Sibirsk. Mat. Zh. 21 (6) (1980), 3-20

[10] V. M. Gordienko, Un problème mixte pour l'équation vectorielle des ondes: Cas de dissipation de l'énergie; Cas mal posés, C. R. Acad. Sci. Paris Sér. A 288 (10) (1979), $547-550$.

[11] —, Symmetrization of a mixed problem for a second order hyperbolic equation with two spatial variables, Sibirsk. Mat. Zh. 22 (2) (1981), 84-104 (in Russian).

[12] D. Hilbert, Über die Darstellung definiter Formen als Summe von Formenquadraten, Math. Ann. 32 (1888), 342-350.

[13] L. Hörmander, Linear Partial Differential Operators, Springer, Berlin 1963.

[14] V. V. Ivanov, Strictly hyperbolic polynomials which do not admit hyperbolic symmetrization, preprint 77, Inst. of Math., Siberian Branch Acad. Sci. USSR, Novosibirsk 1984. 
[15] V. I. Kostin, Transformation of a hyperbolic equation to a symmetric system, Ph.D. thesis, Novosibirsk 1981 (in Russian).

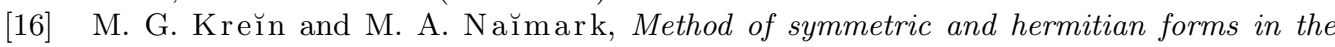
theory of separating roots of algebraic equations, Khar'kov 1936.

[17] J. Leray, Lectures on Hyperbolic Equations with Variable Coefficients, Inst. for Adv. Study, Princeton 1952

[18] N. G. Marchuk, On the existence of solutions of a mixed problem for the vector-valued wave equation, Dokl. Akad. Nauk SSSR 252 (3) (1980), 546-550 (in Russian).

[19] T. Yu. Mikhailova, Symmetrization of invariant hyperbolic equations, ibid. 270 (3) (1983), 246-250 (in Russian).

[20] M. Rosenblatt, A multidimensional prediction problem, Ark. Mat. 3 (5) (1958), 407-424.

[21] A. V. Tishchenko, On a basis of solutions of the homogeneous Hörmander identity, Sibirsk. Mat. Zh. 26 (1) (1985), 150-158 (in Russian). 